\title{
Basic Fibroblast Growth Factor Stimulates Endothelial Regrowth and Proliferation in Denuded Arteries
}

\author{
Volkhard Lindner, ${ }^{*}$ Richard A. Majack, ${ }^{\ddagger}$ and Michael A. Reidy \\ "University of Washington, School of Medicine, Department of Pathology SJ-60, Seattle, Washington 98195; \\ and ${ }^{\ddagger}$ Synergen, Inc., Boulder, Colorado 80301
}

\begin{abstract}
A large percentage of vascular reconstructions, endarterectomies, and angioplasties fail postoperatively due to thrombosis and restenosis. Many of these failures are thought to result from an inability of the vascular endothelium to adequately regenerate and cover the denuded area. After balloon catheter denudation of the rat carotid artery, regrowth of endothelium ceases after $\sim 6 \mathrm{wk}$, leaving a large area devoid of endothelium. Here we show that this cessation of reendothelialization can be overcome by the systemic administration of basic fibroblast growth factor (bFGF). Administration of $120 \mu \mathrm{g}$ bFGF over an 8-h period caused a highly significant increase in the replication rate of endothelial cells at the leading edge of $\mathbf{3 8 . 5}$ vs. $2.1 \%$ in controls, and, when given over a longer period of time (12 $\mu \mathrm{g}$ daily for $12 \mathrm{~d}$ ), resulted in a significant increase in the extent of endothelial outgrowth onto the denuded surface. Furthermore, total regrowth could be achieved within 10 wk after balloon catheter denudation when $12 \mu \mathrm{g}$ bFGF was injected twice per week for a period of 8 wk. Endothelium in unmanipulated arteries responded to bFGF with a significant increase in replication, but no increase in endothelial cell density was observed in these arteries. These data demonstrate that bFGF can act as a potent mitogen for vascular endothelial cells in vivo, and add considerably to our understanding of the mechanism underlying endothelial repair after in vivo vascular injuries. (J. Clin. Invest. 1990. 85:2004-2008.) basic fibroblast growth factor • endothelial regeneration • arterial denudation
\end{abstract}

\section{Introduction}

A unique feature of endothelial cells is that they grow as a monolayer and are subject to growth inhibition by contact with like cells. The precise details of cell contact inhibition are not clear, but disruption of these contacts rapidly initiates cell replication (1-3). Once cell contacts are reformed cell replication stops. In vivo, however, we and others observed that after widespread endothelial loss in arteries the endothelium stops

Address correspondence to Dr. Volkhard Lindner, University of Washington, School of Medicine, Department of Pathology SJ-60, Seattle, WA 98195.

Received for publication 27 December 1989 and in revised form 6 March 1990.

J. Clin. Invest.

(c) The American Society for Clinical Investigation, Inc.

0021-9738/90/06/2004/05 $\$ 2.00$

Volume 85, June 1990, 2004-2008 its regrowth even though reendothelialization is far from complete $(1,4)$. This lack of endothelial regrowth is thought to contribute to the failure of a variety of vascular prostheses (5-9), endarterectomies, and angioplasties (10-13).

A major thrust of this laboratory has been to understand why endothelial cells should spontaneously cease replicating when contact inhibition has not been achieved. We have found that endothelial cells in long-term denuded arteries were capable of further replication (1), and that deletion of the underlying smooth muscle cells (SMC) ${ }^{1}$ did not permit complete reendothelialization (8). These findings suggested that endothelial cells had not senesced and that the underlying SMC were not actively inhibiting their growth. One reason why endothelial cell growth should spontaneously stop came from a recent study where we observed that endothelial cell replication was always associated with immunolocalization of basic fibroblast growth factor (bFGF) in the monolayer, and when endothelial cells spontaneously ceased replicating no bFGF could be detected (14). This raised the possibility that bFGF might play a major role in the control of endothelial cell growth in vivo. In this report we show that endothelial replication in denuded rat arteries can be significantly restimulated by systemic administration of bFGF and that the administration of this mitogen also leads to a significant increase in endothelial cell coverage on the denuded artery.

\section{Methods}

A total of 54 male 3-4-mo-old Sprague-Dawley rats were used (Tyler Laboratories, Bellvue, WA). All surgery and infusions were performed under general anesthesia with an initial intramuscular injection of 0.06 $\mathrm{mg} / \mathrm{kg}$ fentanyl (Innovar-Vet; Pitman-Moore, NJ) and additional injections when necessary. Anesthesia with ether was chosen for tail vein injections.

bFGF infusion experiments. These experiments were carried out on rat carotid arteries at 4 and $6 \mathrm{wk}$ after balloon catheter denudation. These times were chosen because we wanted to test whether bFGF would increase a markedly slowed endothelial cell replication (4 wk after denudation) or whether it would restimulate cell replication in endothelial cells that had nearly ceased replicating (i.e., 6 wk after denudation).

The left carotid artery was denuded of endothelium with a $2 \mathrm{~F}$ Fogarty balloon catheter (American V. Mueller, Irvine, CA) (1) and 4 wk later bFGF was infused. The recombinant human bFGF (Synergen, Inc., Boulder, $\mathrm{CO}$ ) used was of pharmaceutical grade delivered in vehicle (0.3 M glycerine, $10 \mathrm{mM}$ sodium phosphate, $\mathrm{pH} 7.3)$ with a

1. Abbreviations used in this paper: bFGF, basic fibroblast growth factor; SMC, smooth muscle cells. 
protein concentration of $1.2 \mathrm{mg} / \mathrm{ml} .120 \mu \mathrm{g}$ of bFGF diluted in $2 \mathrm{ml}$ of saline was infused per animal. Control infusions contained equal amounts of vehicle diluted in $2 \mathrm{ml}$ of saline. The infusion was started with a bolus injection of $30 \mu \mathrm{g}$ bFGF given intraarterially, followed by a continuous infusion over an 8-h period through a catheter placed into the aortic arch via the right axillar artery. The remainder of the bFGF $(90 \mu \mathrm{g})$ was administered over an 8 -h time period. In control animals equal amounts of vehicle were infused in the same fashion. At 24 and $32 \mathrm{~h}$ after starting the infusion the animals were injected with tritiated thymidine $(50 \mu \mathrm{Ci} / 100 \mathrm{~g}$ body weight, $6.7 \mathrm{mCi} / \mathrm{mM}$; New England Nuclear, Boston, MA). 1 h before killing Evans blue $(0.5 \mathrm{ml}$, $5 \%$ in saline) was injected intravenously to mark the still denuded surface and the animals were perfused-fixed $33 \mathrm{~h}$ after the start of the $\mathrm{bFGF}$ infusion. The technique for perfuse-fixing arteries at physiological flow and pressure has been described elsewhere (14). Briefly, the rats were killed with an overdose of sodium pentobarbital, a catheter was placed in the abdominal aorta, and the animals were fixed with $4 \%$ paraformaldehyde in phosphate buffer $(0.1 \mathrm{M}, \mathrm{pH} 7.3)$ at physiologic pressure and flow for $4 \mathrm{~min}$. Tissue of the proximal and distal carotid artery that contained the endothelial-SMC interfaces was processed for en face preparation as described below.

The identical experiment was carried out in groups of animals $6 \mathrm{wk}$ after their left carotid artery had been denuded of endothelium with a balloon catheter. Endothelial replication in these vessels was measured as described above.

Endothelial cell outgrowth. In the first experiment we asked whether prolonged administration of bFGF could cause an increase in endothelial outgrowth. Animals had the left common carotid denuded of endothelium. $6 \mathrm{wk}$ later (when reendothelialization had nearly ceased) the animals received injections of either $12 \mu \mathrm{g}$ bFGF or an equal amount of vehicle via tail vein daily for $12 \mathrm{~d}$. The amount of endothelial regrowth in the left carotid arteries was measured macroscopically using Evans blue staining to mark the still denuded area. The reference points for total endothelial outgrowth were the common carotid bifurcation and the aorta-carotid junction.

A second experiment was designed to investigate whether total endothelial regrowth could be achieved. Two groups of rats had the left carotid denuded of endothelium and 2 wk later $12 \mu \mathrm{g}$ of bFGF (or vehicle) was injected intravenously twice a week for a period of $8 \mathrm{wk}$. We chose 2 wk after denudation for the start of the bFGF administration because before this time spontaneous endothelial replication was high (14). $10 \mathrm{wk}$ after denudation the extent of endothelial outgrowth was measured.

Time course of endothelial replication after $b F G F$ injection. For this experiment groups of four animals had the left carotid artery denuded of endothelium $6 \mathrm{wk}$ before the injection of either $30 \mu \mathrm{g}$ bFGF or a corresponding vehicle injection into the tail vein. The animals in these groups were killed 2, 4, and $7 \mathrm{~d}$ after the injection. They were injected with $\left[{ }^{3} \mathrm{H}\right]$ thymidine 1,9 , and $17 \mathrm{~h}$ before death. Replication indices for the endothelium were obtained as described below.

Unmanipulated endothelium after bFGF injection. The effect of bFGF of unmanipulated endothelium was investigated on Häutchen preparations of the thoracic aorta from the experiments mentioned above. In addition, in one experiment $12 \mu \mathrm{g}$ of bFGF was injected into animals via the tail vein and the replication index of uninjured endothelium was measured. Labeling with three doses of $\left[{ }^{3} \mathrm{H}\right]$ thymidine 24 , 32 , and $40 \mathrm{~h}$ after the injection was performed as described above.

Immunocytochemistry on en face preparations. For all studies where endothelial replication was quantitated the luminal surface of all carotid arteries was stained with an antibody against Factor VIII-related antigen to facilitate the differentiation between luminal SMC and endothelial cells. The excised carotids were cut open longitudinally and pinned out on Teflon cards. Methanol permeabilization of the tissue and blocking of endogenous peroxidase with $0.3 \%$ hydrogen peroxide incubation for $30 \mathrm{~min}$ was performed as previously described (15). Nonspecific binding of rabbit IgG was prevented by preincubating the tissue with $2 \%$ normal goat serum. A rabbit antibody (IgG) against human Factor VIII-related antigen (Dako Corp., Santa Bar- bara, CA) was used at a concentration of 1:1,000. This tissue was then incubated with biotinylated goat anti-rabbit IgG (Vector Laboratories, Inc., Burlingame, CA) followed by peroxidase labeling with an avidin-peroxidase complex (ABC Kit; Vector Laboratories, Inc.). All incubations lasted $30 \mathrm{~min}$ and were carried out at room temperature. To visualize the antigen-antibody complexes, the tissue was incubated with 0.05\% 3,3'-diaminobenzidine (Sigma Chemical Co., St. Louis, $\mathrm{MO}$ ) in $0.05 \mathrm{M}$ Tris-HCI, $\mathrm{pH} 7.6$, with $0.003 \%$ hydrogen peroxide for 4 min. Häutchen preparations of the tissue were made according to the procedure described by Schwartz and Benditt (16). The preparations were counterstained with hematoxylin. In all cases, corresponding concentrations of either nonimmune rabbit IgG or serum were included as controls.

Autoradiography. For autoradiography, the preparations were dipped in Kodak NTB-2 emulsion and exposed for 2 wk. The slides were then developed, stained with hematoxylin, and mounted under coverslips. For en face preparations the total number of endothelial cells and the number of labeled endothelial cells were counted using a square-shaped reticule in a $10 \times$ eyepiece with a $40 \times$ objective. Quantitation of replicating endothelial cells was restricted to the area that was within $1 \mathrm{~mm}$ of the interface with the luminal SMC, equivalent to four reticule fields deep in the endothelial monolayer.

\section{Results}

Endothelial replication. The presence of bFGF in endothelial cells appears to correlate well with their replication index (14). This became apparent in a previous study where we observed that in chronically denuded arteries those endothelial cells that spontaneously stop proliferating stain very weakly for bFGF, whereas rapidly replicating endothelium stained strongly (14). This suggested to us that bFGF synthesis might play an important role in endothelial cell replication in vivo.

To investigate the relationship between cessation of endothelial cell replication and bFGF we determined whether endothelial cells in chronically denuded arteries which have stopped replicating can be restimulated by the administration of exogenous bFGF. In the studies described below, bFGF was administered to animals at both 4 and 6 wk after denuding injury to the carotid arteries. $4 \mathrm{wk}$ after denudation endothelial cell replication markedly slowed down and therefore any increase in replication in response to exogenous bFGF might be easily detected. In this experiment bFGF was given to animals $4 \mathrm{wk}$ after their carotid artery was denuded of endothelium by a balloon catheter. By autoradiography, the replication index of endothelium at the leading edge, which can be readily visualized by staining with an antibody against Factor VIII-related antigen, was found to be significantly increased after an 8-h infusion of bFGF (Fig. 1,a and $b$ ). The replication rate 4 wk after denuding injury was increased from $4.95 \%$ in controls to $30.11 \%$ after bFGF treatment (Fig. 2).

Another experiment was designed to investigate whether bFGF could restimulate endothelial cells that have become quiescent. In this experiment animals had the left carotid artery denuded of endothelium with a balloon catheter and left for $6 \mathrm{wk}$. At this time the replication rate of the leading endothelial cells was approximately equal to that observed in cells of uninjured arteries. As shown in Fig. 2, infusion of bFGF into these animals again caused a highly significant increase in endothelial replication (38.49\%), whereas with infusion of vehicle alone the replication rate was $2.08 \%$. In unmanipulated control arteries bFGF caused a statistically significant increase in endothelial cell replication (Fig. 2) as compared with arteries that received vehicle alone, but this increase was markedly 

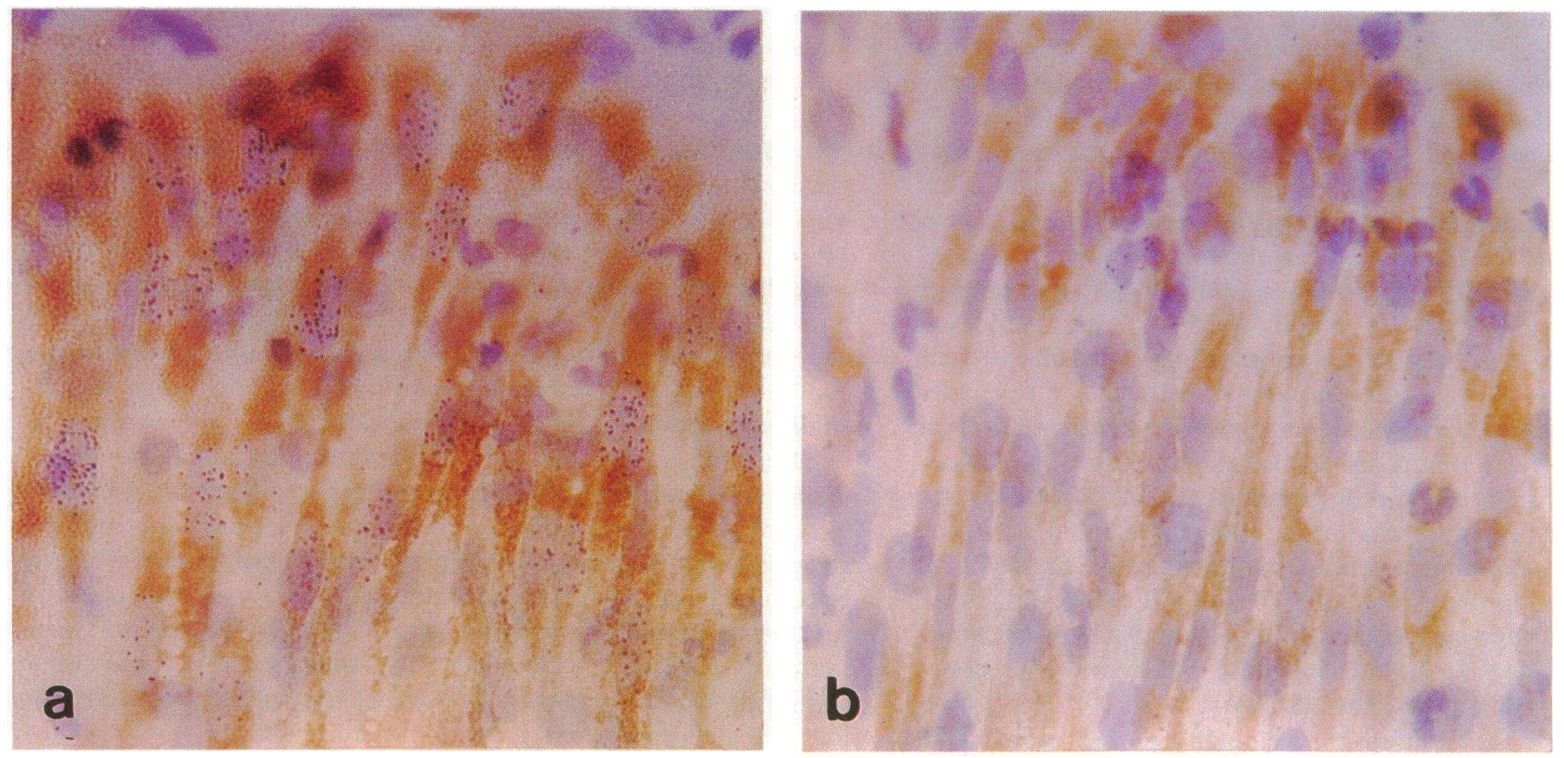

Figure 1. Photomicrograph of en face preparation of endothelial cells at leading edge of denuded artery 6 wk after injury. To facilitate the identification of endothelial cells, the tissue was stained for Factor VIII-related antigen. $a$, After bFGF infusion numerous replicating cells were observed as indicated by the presence of silver grains over the nuclei $(\times 400)$. $b$, After vehicle infusion no replicating cells were seen $(\times 400)$.

smaller than that observed in the still denuded arteries. The above data clearly demonstrate that bFGF causes a very significant increase in the replication rate of recently regenerated endothelium in arteries that are still partially denuded.

Endothelial outgrowth. An increase in endothelial cell replication by itself does not necessarily imply increased endothe-

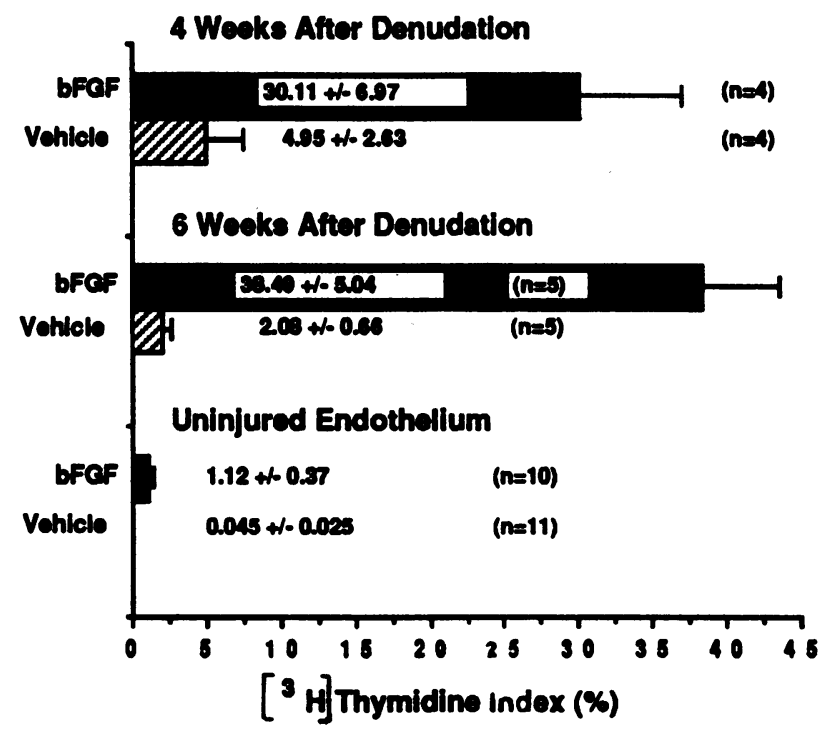

Figure 2. Endothelial cell replication after bFGF and control infusion. 4 and 6 wk after balloon catheter denudation $120 \mu \mathrm{g}$ of bFGF was infused intraarterially over $8 \mathrm{~h}$. The $\left[{ }^{3} \mathrm{H}\right]$ thymidine index was calculated from autoradiographs of en face preparations of the proximal and distal endothelial cell sheets adjacent to the denuded surface. Endothelial cell replication 4 and 6 wk after injury was signifcantly higher in the bFGF-treated animals $(P \leq 0.0001)$. Replication rates of uninjured carotid endothelium were significantly higher in the bFGF-treated animals $(P \leq 0.006)$. Data represent means \pm SEM; $n=$ number of animals in each group. lial outgrowth since injurious agents such as endotoxin or mechanical injuries can also initiate an increase in endothelial cell replication but do not stimulate regrowth of endothelium onto the denuded surface (15). To test whether the bFGF-mediated increase in endothelial replication would result in increased outgrowth of the endothelium, the following experiment was performed. Carotid arteries were denuded with a balloon catheter and 6 wk later the animals received multiple doses of bFGF. Daily administration of bFGF $(12 \mu \mathrm{g})$ for $12 \mathrm{~d}$ caused a
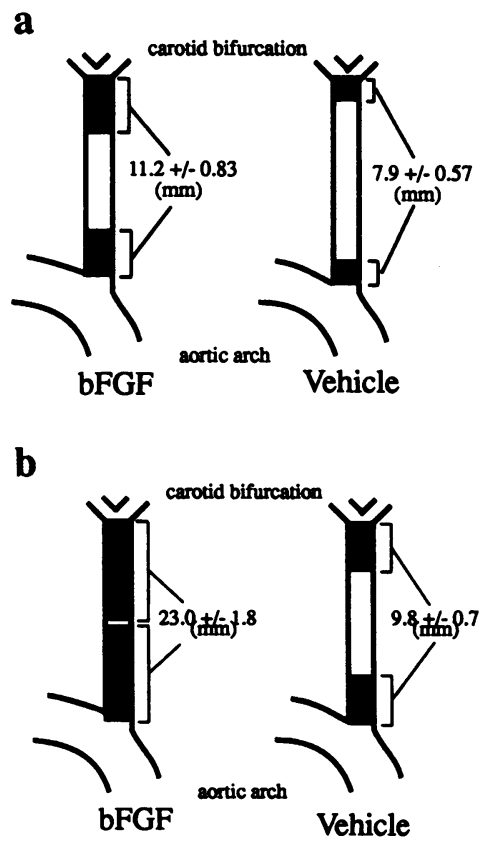

Figure 3. Endothelial regrowth in the common artery of the rat. $a, 6 \mathrm{w}$ after balloon catheter denudation of the left carotid artery $12 \mu \mathrm{g}$ of bFGF in 0.2 $\mathrm{ml}$ saline or an equal amount of vehicle in saline was injected intravenously via the tail vein once daily over a period of $12 \mathrm{~d}$. Regrowth occurring from the aortic arch and the bifurcation was measured after the treatment period. Significantly more endothelial regrowth occurred in the bFGF-treated animals $(P$ $\leq 0.013, n=6$ animals in each group). $b, 2 \mathrm{wk}$ after denudation $12 \mu \mathrm{g}$ of bFGF was injected every $4 \mathrm{~d}$ over a period of $8 \mathrm{wk}$. In one animal regrowth was complete $(P \leq 0.002, n=3$ animals in each group). Data represent means of total regrowth \pm SEM. 
significant increase in the area populated by new endothelial cells as compared with controls that received vehicle alone (Fig. $3 a$ ).

This significant increase in outgrowth prompted us to test whether total endothelial regrowth could be achieved by these means. In the following study the administration of bFGF was started 2 wk after denudation, which is when the spontaneous growth of endothelium has started to decline. Thus, 2 wk after balloon catheter denudation of the carotid artery, bFGF was injected for $8 \mathrm{wk}$ ( $12 \mu \mathrm{g}$ every $4 \mathrm{~d}$ ). In one animal the endothelium had regrown to completion and in the others $<2.0 \mathrm{~mm}$ of denuded area remained (Fig. $3 \mathrm{~b}$ ). In the corresponding control group receiving vehicle alone for $8 \mathrm{wk}$ the area still denuded of endothelium measured $12.3 \pm 1.8 \mathrm{~mm}$ (SEM). Thus addition of systemic bFGF not only caused a dramatic increase in the replication of those endothelial cells but also stimulated their growth onto a denuded surface, which before the start of the experiment appeared to abrogate coverage by endothelium.

Endothelial cell density. Since bFGF stimulated quiescent endothelium of unmanipulated arteries we next asked whether multiple administrations of bFGF would cause an increase in the number of endothelial cells in these arteries. When $12 \mu \mathrm{g}$ of bFGF was administered daily to animals with an unmanipulated aorta for a period of $12 \mathrm{~d}$, the endothelial cell densities of the thoracic aortae (measured by counting the number of endothelial cells per high power field) were not significantly different $\left(2,024\right.$ cells $/ \mathrm{mm}^{2}$ in the bFGF group and 2,011 cells/ $\mathrm{mm}^{2}$ in the control group). This regimen of bFGF, however, significantly increased endothelial cell replication (Fig. 4).

Time course of endothelial replication. Since exogenous bFGF restored the ability of the endothelium to replicate, one possibility was that these cells had resumed their normal function and so were able to spontaneously proliferate after a single exogenous stimulus. We approached this issue by asking if endothelial cell replication was chronically elevated after a single administration of bFGF. Groups of animals were killed at various times after a single intravenous injection of bFGF. As can be seen in Fig. 5, the replication index fell with time, and $7 \mathrm{~d}$ after the bFGF injection no increased replication was observed.

\section{Discussion}

Previous studies from this laboratory have shown that in vivo, endothelial cells have a very limited capacity for repopulating denuded areas (1). This has been found to occur not only in

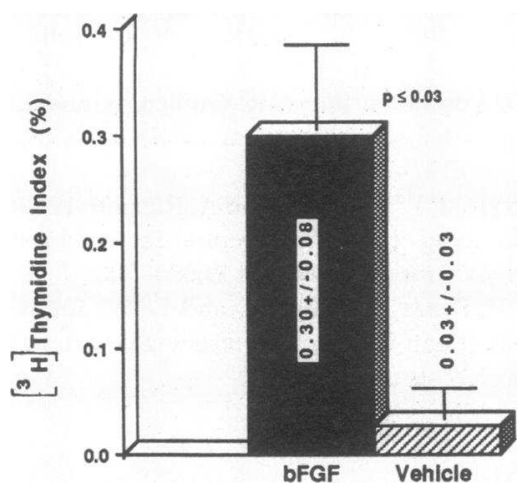

Figure 4. Replication index of unmanipulated aortic endothelium $2 \mathrm{~d}$ after intravenous injection of $12 \mu \mathrm{g}$ of bFGF. Data represent means \pm SEM; $n=3$ animals in each group.

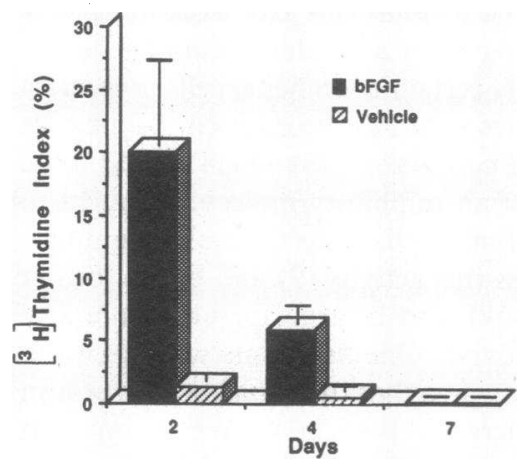

injured arteries $(1,4,17)$ but also in vascular grafts $(6,8)$ where the inability of the endothelium to repopulate the lumen is thought to jeopardize graft patency. Thus far no procedures have been successful in promoting endothelial outgrowth onto the denuded surface once the endothelial cells at the leading edge ceased replicating. In a recent in vivo study we observed that replicating endothelial cells stain strongly with an antibody against bFGF and that quiescent endothelium shows only little staining (14). A lack of bFGF was also observed in those endothelial cells at the leading edge of chronically denuded arteries which had ceased replicating. This suggested to us that bFGF may play a key role in the control of endothelial cell replication in vivo. The aim of this study, therefore, was to investigate whether cessation of endothelial growth, which seems to be associated with a lack of endogenous bFGF, could be overcome by the administration of exogenous bFGF.

The data reported here show that the cessation of endothelial cell growth observed after widespread denudation is not irreversible and that addition of bFGF leads to a very dramatic and significant increase in cell replication as well as an increase in the area repopulated with new endothelium. bFGF is known to be a potent mitogen for endothelial cells in vitro $(18-21)$ and several investigators have shown that bFGF is angiogenic in a variety of in vivo and in vitro models $(19,22$, 23). However, the direct effects of this growth factor on the endothelium of large vessels in vivo have not been studied. The data from this study show that not only is bFGF active on vascular wall cells when administered in vivo, but that it is a potent mitogen for large vessel endothelium. Indeed, the endothelial cell replication rates induced by bFGF approximated those rates observed in arteries soon after injury when regrowth is at its maximum. Interestingly, however, the same dose of bFGF had a markedly different response on the endothelium of uninjured arteries. The replication index of these cells was significantly increased $(1.12 \%)$ as compared with controls $(0.045 \%)$, but this increase was $\sim 30$-fold lower than the increase observed in endothelial cells from denuded arteries. Since all the endothelial cells are likely to be exposed to the same concentration of circulating bFGF, other factors must be modulating the process. One possibility is that the number of bFGF receptors varies between these two cell populations and that the endothelial cells on the denuded arteries bind more bFGF. Little is known about what factors might control bFGF receptor expression, but of interest is the report that receptor 
number is high when cell density is low (24), and the endothelial cells at the leading edge would be considered a zone of low density. If this was true then the lack of availability of bFGF to the endothelium would be a dominant fact explaining why these cells stop replicating soon after denudation. Alternatively, the reason why only certain endothelial cells proliferate at such relatively low rates could be because confluent cells have extensive junctional complexes (25) which appear to be related to the presence of an inhibitory molecule thought to control their growth (2). Endothelial cells from an incomplete monolayer do not express this activity (2) and have a higher replication rate. This could also be true for an incomplete endothelial monolayer in vivo. One final issue with regard to the uninjured endothelium was that there was no increase in cell density despite the increased replication. We assume that this indicates an increase in cell turnover which has also been found in vascular endothelium under other circumstances (15).

In this study we have demonstrated that systemic administration of bFGF can significantly restimulate quiescent endothelial cells in an incomplete endothelial monolayer in a denuded artery. Furthermore, continuous administration of bFGF leads to a significant increase in the area of reendothelialization and complete regrowth can be achieved. The significance of these data is twofold: first, we show that bFGF is a potent mitogen for large vessel endothelium in vivo; second, and more important, we demonstrate that quiescent endothelial cells can be restimulated by bFGF to grow onto denuded areas where reendothelialization would not normally occur. One possible implication of this result is that complete reendothelialization of synthetic vascular grafts might be achieved after bFGF treatment. It is generally believed that a complete endothelial covering may significantly extend the lifespan of these prostheses and studies are now testing the feasibility of this approach.

\section{Acknowledgments}

We wish to thank Abel Eng, Eric Olson, Stella Chao, and Colleen Irvin for their skilled technical assistance.

This work was supported by the Deutsche Forschungsgemeinschaft through a fellowship awarded to Dr. Volkhard Lindner (Li 429/1-1) and National Institutes of Health grants HL-03174 and HL-41103.

\section{References}

1. Reidy, M. A., A. W. Clowes, and S. M. Schwartz. 1983. Endothelial regeneration. V. Inhibition of endothelial regrowth in arteries of rat and rabbit. Lab. Invest. 49:569-575.

2. Heimark, R. R., and S. M. Schwartz. 1985. The role of membrane-membrane interactions in the regulation of endothelial cell growth. J. Cell Biol. 100:1934-1940.

3. Reidy, M. A., 1985. Biology of disease. A reassessment of endothelial injury and arterial lesion formation. Lab. Invest. 53:513-520.

4. Goff, S. G., H. D. Wu, L. R. Sauvage, Y. Usui, A. R. Wechezak, D. E. Coan, R. E. Arnell, and M. W. Walker. 1988. Differences in reendothelialization after balloon catheter removal of endothelial cells, superficial endarterectomy, and deep endarterectomy. J. Vasc. Surg. 7:119-129.

5. Allen, B. T., J. A. Long, R. E. Clark, G. A. Sicard, K. T. Hopkins, and M. J. Welch. 1984. Influence of endothelial cell seeding on platelet deposition and patency in small-diameter Dacron arterial grafts. $J$. Vasc. Surg. 1:224-233.

6. Berger, K., L. R. Sauvage, A. M. Rao, and S. J. Wood. 1972. Healing of arterial prostheses in man: its incompleteness. Ann. Surg. 175:118-127.

7. Harker, L. A., S. J. Slichter, and L. R. Sauvage. 1977, Platelet consumption by arterial prostheses: the effect of endothelialization and pharmacologic inhibition of platelet function. Ann. Surg. 186:594601.

8. Reidy, M. A. 1988. Endothelial regeneration. VIII. Interaction of smooth muscle cells with endothelial regrowth. Lab. Invest. 59:36-43.

9. Sauvage, L. R., K. E. Berger, S. J. Wood, S. G. Yates, J. C. Smith, and P. B. Mansfield. 1974. Interspecies healing of porous arterial prostheses. Arch. Surg. 109:698-705.

10. Cossman, D., A. D. Callow, A. Stein, and G. Matsumato. 1978. Early restenosis after carotid endarterectomy. Arch. Surg. 113:275278.

11. French, B. N., and N. B. Rewcastle. 1977. Recurrent restenosis at site of carotid endarterectomy. Stroke. 8:597-605.

12. Fuster, V., and J. H. Chesebro. 1986. Role of platelets and platelet inhibitors in aortocoronary artery vein-graft disease. Circulation. 73:227-232.

13. Unni, K. K., B. A. Kottke, J. L. Titus, R. L. Frye, R. B. Wallace, and A. L. Brown. 1974. Pathologic changes in aortocoronary saphenous vein grafts. Am. J. Cardiol. 34:526-532.

14. Lindner, V., M. A. Reidy, and J. Fingerie. 1989. Regrowth of arterial endothelium: denudation with minimal trauma leads to complete endothelial cell regrowth. Lab. Invest. 61:556-563.

15. Reidy, M. A., M. Chopek, S. Chao, T. McDonald, and S. M. Schwartz. 1989. Injury induces increase in von Willebrand factor in rat endothelial cells. Am. J. Pathol. 134:857-864.

16. Schwartz, S. M., and E. P. Benditt. 1973. Cell replication in the aortic endothelium: a new method for study of the problem. Lab. Invest. 28:699-707.

17. Reidy, M. A., D. Standaert, and S. M. Schwartz. 1982. Inhibition of endothelial cell regrowth: cessation of aortic endothelial cell replication after balloon catheter denudation. Arteriosclerosis. 2:216220.

18. Baird, A., F. Esch, P. Mormède, N. Ueno, N. Ling, P. Böhlen, S.-Y. Ying, W. B. Wehrenberg, and R. Guillemin. 1986. Molecular characterization of fibroblast growth factor: distribution and biological activities in various tissues. Recent Prog. Horm. Res. 42:143-205.

19. Folkman, J., and M. Klagsbrun. 1987. Angiogenic factors. Science (Wash. DC). 235:442-447.

20. Gospodarowicz, D., G. Neufeld, and L. Schweigerer. 1986. Molecular and biological characterization of fibroblast growth factor, an angiogenic factor which also controls the proliferation and differentiation of mesoderm and neuroectoderm derived cells. Cell Differ. 19:1-17.

21. Gospodarowicz, D., G. Neufeld, and L. Schweigerer. 1986. Fibroblast growth factor: review. Mol. Cell. Endocrinol. 46:187-204.

22. Hayek, A., F. L. Culler, G. M. Bearrie, A. D. Lopez, P. Cuevas, and A. Baird. 1987. In vivo model for study of the angiogenic effects of basic fibroblast growth factor. Biochem. Biophys. Res. Commun. 147:876-880.

23. Montesano, R., J. D. Vasalli, A. Baird, R. Guillemin, and L. Orci. 1986. Basic fibroblast growth factor induces angiogenesis in vitro. Proc. Natl. Acad. Sci. USA. 83:7297-7301.

24. Veomett, G., C. Kuszynski, P. Kazakoff, and A. Rizzino. 1989. Cell density regulates the number of cell surface receptors for fibroblast growth factor. Biochem. Biophys. Res. Commun. 159:694-700.

25. Spagnoli, L. G., G. P. Pietra, S. Villaschi, and L. W. Johns. 1982. Morphometric analysis of gap junctions in regenerated arterial endothelium. Lab. Invest. 46:139-148. 